\title{
Total Dissolved Solids in Water as a Function of Growing Percent Impervious Surface Cover in the Watershed Suggest Pitfalls in Growth of Infrastructure
}

\author{
Jason Kong', Matthew Bingham² \\ ${ }^{1}$ Milton Academy, 170 Centre Street, Milton, MA 02186 \\ ${ }^{2}$ Milton Academy Science Department, Milton Academy, 170 Centre Street, Milton, MA 02186
}

\begin{abstract}
The spread of globalization and infrastructure can pose a threat to the quality of water that surrounding ecosystems depend on to live. Impervious surfaces, such as roads, buildings, sidewalks, and parking lots, characterize this insidious proliferation of water quality degradation. Impervious surfaces contribute to heightened levels in floods, runoff, water pollution, erosion, and many other threats to the natural environment. To further investigate the relationship between the percent impervious surface cover in a watershed and the water quality of the water, this experiment was conducted along Pine Tree Brook, a stream in the Neponset River Watershed of Massachusetts. At eight evenly spaced out locations along the brook, water samples were taken as we travelled downstream from the source of Pine Tree Brook in the Blue Hills to the end of the brook in the town of Milton, MA. These samples were then measured for their water quality in total dissolved solids. Computer software was then used to calculate the percent impervious surface cover in the watershed for each respective water-sampling site. We observed that as the percent impervious surface cover increased, the total dissolved solids in the water increased as well. These results suggest that the increased infrastructure can possibly have adverse effects on the water quality in the surrounding watershed. Despite the uncertainty in the demarcation of the watershed and the confounding variable of pollution-causing agents, this experiment displays a moderately positive trend between impervious surface cover and water contamination. These results suggest that an improved experimental methodology will be able to suggest more profoundly the effect infrastructure has on the surrounding aquatic environment.
\end{abstract}

Keywords: Impervious Surfaces, Water Quality, Infrastructure, Watershed, Pollution, Climate Change

\section{INTRODUCTION}

Globalization pushes the growth of infrastructure. Manufacturing requires more buildings. Population calls for more houses. Transportation demands increasing road networks. The consequences of these four current issues elucidate the fact that impervious surfaces have begun to take over the natural surface of the Earth. Natural surfaces that allow rainwater to be reabsorbed into the soil have been blanketed with roads, parking lots, and buildings. This inhibition of rainwater infiltration leads to increased storm water runoff (Center for Watershed Protection, 1998). Not only does this water cause the surrounding watershed to rapidly fill with water, but also this fast flowing water can pick up and carry more sediment and other pollutants, such as heavy metals, grease, and oils, into reservoirs (Beckwith, 2007). These pollutants threaten the ecosystems within the streams and other bodies of water in the surrounding watershed (Hill, 2009). Fish and plant life suffer from augmented contamination levels.

As species of aquatic life perish from the pollution, the stream biodiversity decreases and the subsequent disasters threaten the food chain (Polycarpou, 2010). Catastrophic floods have also resulted from large quantities of water not being able to infiltrate the ground (Polycarpou, 2010). This increased water flow and heightened flood peak lead to more erosion along the streambeds as well (Clean Water Education Partnership, 2003). As erosion terrorizes the trees and vegetation that grow along the banks of rivers, ecosystems are further harmed. Plants that provide shelter and food for the animals will be washed away (Center for Watershed Protection, 1998). 
Jason Kong \& Matthew Bingham "Total Dissolved Solids in Water as a Function of Growing Percent Impervious Surface Cover in the Watershed Suggest Pitfalls in Growth of Infrastructure?"

Water pollution not only affects animal life, but that of humans as well. As sometimes the bulk of a population's drinking water comes from nearby bodies of water, the health of the surrounding families can be adversely affected (Hill, 2009). Taking these negative affects into consideration, this experiment seeks to provide a more robust understanding of current water quality in Milton, MA.

This experiment was conducted at eight locations along Pine Tree Brook, a stream in the Neponset River Watershed (see Fig. 1). Moving downstream from the source of Pine Tree Brook (Chestnut Run, Milton, MA) to the end of the brook at (Central Avenue, Milton, MA), water quality data were recorded on-site at the respective locations. Percentages of impervious surface coverage in the specific watershed surrounding each location were calculated using a computer imaging software and a GIS. The eight Pine Tree Brook locations also exhibited a gradient from a more rural area in the Blue Hills to a medium-density residential area with large infrastructure and paved roads in the town of Milton. The water quality data relied on the measurement of total dissolved solids (TDS) in the water. TDS takes into account chemicals, organic materials, and inorganic materials that are dissolved into the water. A high TDS reading can render a certain sample of water harmful (Oram, 2014). TDS does not directly correlate with harmful substances in the water, but within the scope of this experiment, pesticides, oils, salts, metals, chemicals, and other harmful contaminants likely present in the suburban storm water runoff are assumed to make up the majority of the substances dissolved into Pine Tree Brook. The purpose of this experiment was to evaluate how the percentage of impervious surface coverage in the surrounding watershed affected the water quality at each location. Because more impervious surface coverage causes more runoff to carry pollution into the water, our hypothesis is that the TDS in the stream will increase as the percentage of impervious surface coverage increases.
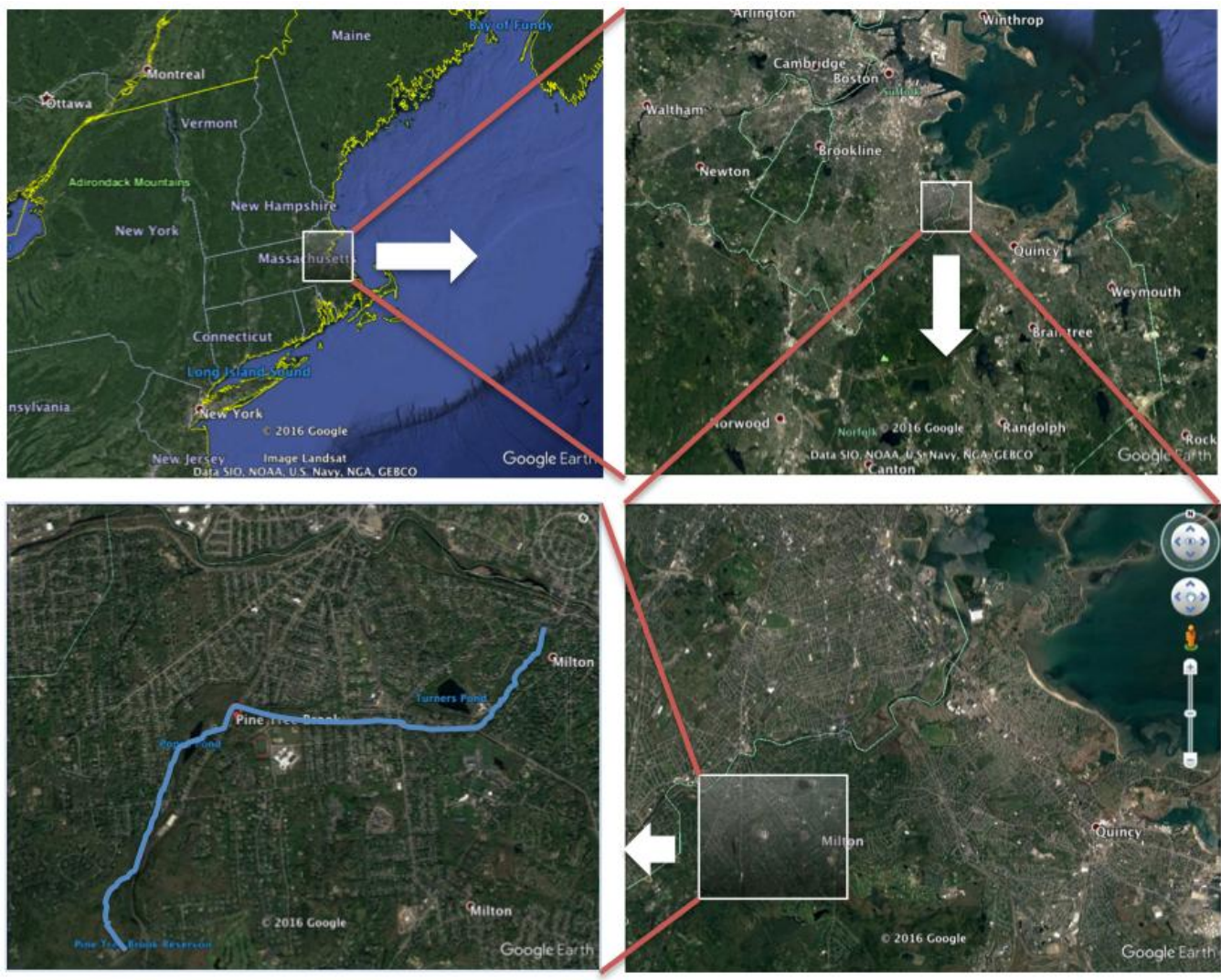

Figure1. Incremental zoom field map of Pine Tree Brook. The blue line indicates Pine Tree Brook.

\section{METHODS}

To test our hypothesis that the TDS in the stream will increase as the percentage of impervious surface coverage increases, we conducted an experiment along Pine Tree Brook, which is part of the Neponset River Watershed. This experiment was also conducted after a day of rainfall so that we could allow the rainwater to run off into the streams and contribute to our data. We wanted to accurately represent the whole of Pine Tree Brook. Beginning in the Blue Hills, the source of Pine Tree Brook, and ending at Central Avenue, the end of Pine Tree Brook, we traveled downstream to eight sites that were separated by roughly the same distance (see Fig. 2). At each location, we collected water samples with a particular focus on the best practice of triple-rinsing the sample 
Jason Kong \& Matthew Bingham "Total Dissolved Solids in Water as a Function of Growing Percent Impervious Surface Cover in the Watershed Suggest Pitfalls in Growth of Infrastructure?"

container to rid the container of past samples that could confound our data. The eight distinct water samples were then brought back to the laboratory. Using an accurately calibrated Vernier LabQuest Conductivity Sensor set to measure in TDS (mg/L), we recorded the TDS data for each water sample. In between the samples, we made sure to thoroughly rinse the conductivity meter with distilled water so that the previous sample would have little influence on the next sample. Next, we used Oliver, an online mapping tool provided by Mass GIS, and Adobe Photoshop. For each location, we transferred a JPEG file of that location with pre-programmed map data layers that displayed impervious surfaces and topographic lines into the Adobe Photoshop imaging software. The impervious surface data layer that Oliver provided was indicated by a solid purple color (see Fig. 3). With Adobe Photoshop, we delineated a watershed for each specific location by drawing a polygon with the selection tool option. The borders were drawn according to the topographic map detail. For each location, we looked at what direction the water would flow due to changes in the elevation and drew the polygon around an area that would sufficiently account for the surrounding area that drains to the stream. Using the pixel counter tool provided by Adobe Photoshop, we recorded the number of pixels in the selected watershed and the number of pixels of impervious surfaces within that specific watershed (see Fig. 3). We set the pixel counter to count all colored pixels to find the watershed pixel count. Then, we set the pixel counter to count only purple pixels to find the impervious surface pixel count. As we analyzed each subsequent downstream location, we took care to add the previous watershed and impervious surface areas to the next pair of results. As we move downstream, both the watershed and impervious surface area increases because water flows into the river from the watershed of previous locations as well as the watershed in the current sampling location. Once we compiled the watershed and impervious surface data for all eight locations, we calculated the percentage of impervious surface coverage for each location. For each of the eight locations, we divided the number of impervious surface pixels by the number of pixels in the whole watershed. We then multiplied the result of that faction by one hundred to find what percentage of the selected watershed was covered by impervious surfaces. For example, site one's watershed data and impervious surface data are calculated for percentages. Then, site two's watershed data, which is site one's specific watershed data added to site two's specific watershed data, and impervious surface data, which is site one's specific impervious surface area added to site two's specific impervious surface areas, are calculated for percentages.
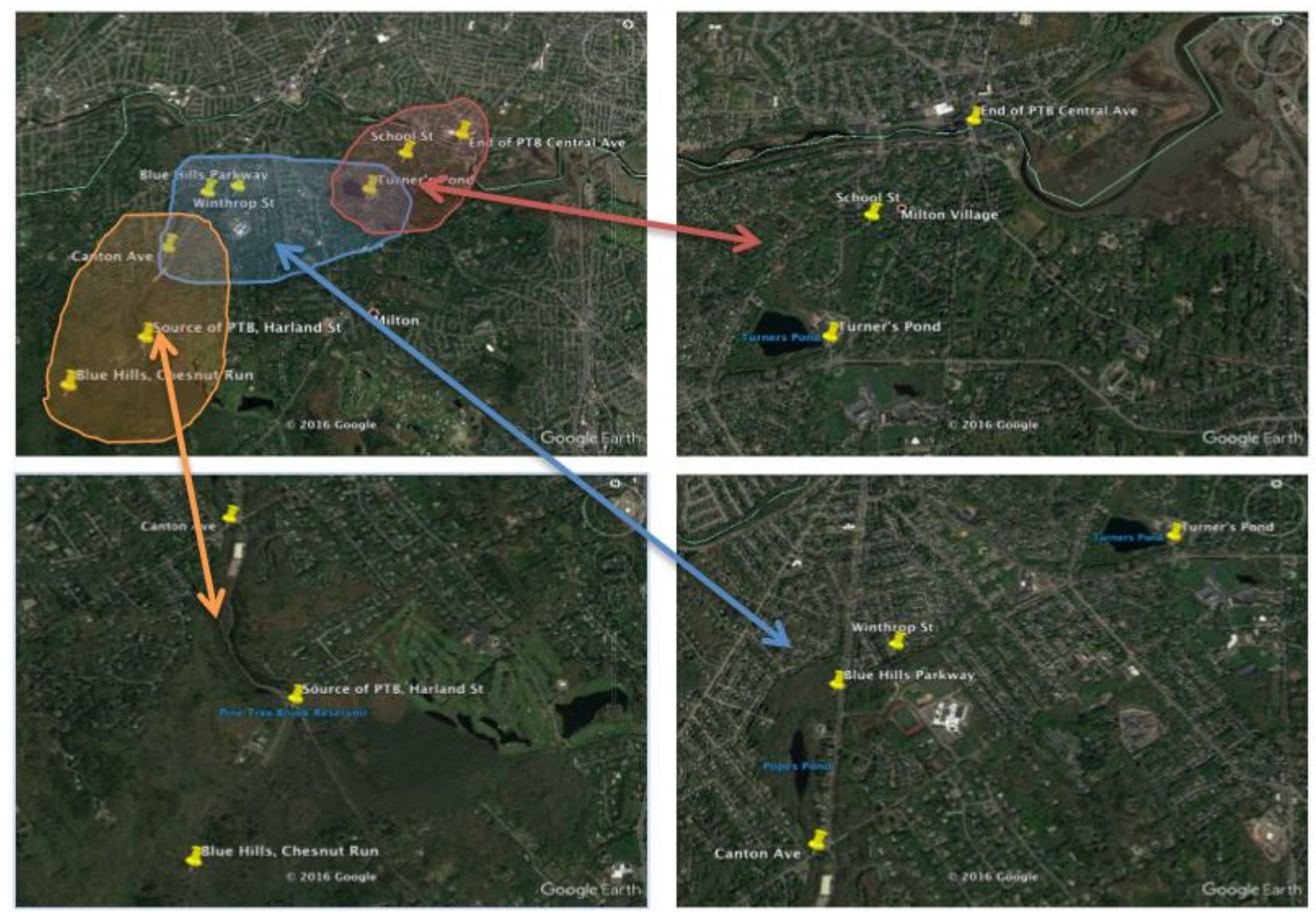

Figure2. Sampling sites along Pine Tree Brook. The map with orange, blue, and red polygons is the cumulative map of all the sites. The orange line directs to a closer perspective of the sites 1, 2, and 3. The blue line directs to a closer perspective of sites 3, 4, 5, and 6. The red line directs to a closer perspective of sites 6, 7, and 8 . 
Jason Kong \& Matthew Bingham "Total Dissolved Solids in Water as a Function of Growing Percent Impervious Surface Cover in the Watershed Suggest Pitfalls in Growth of Infrastructure?"

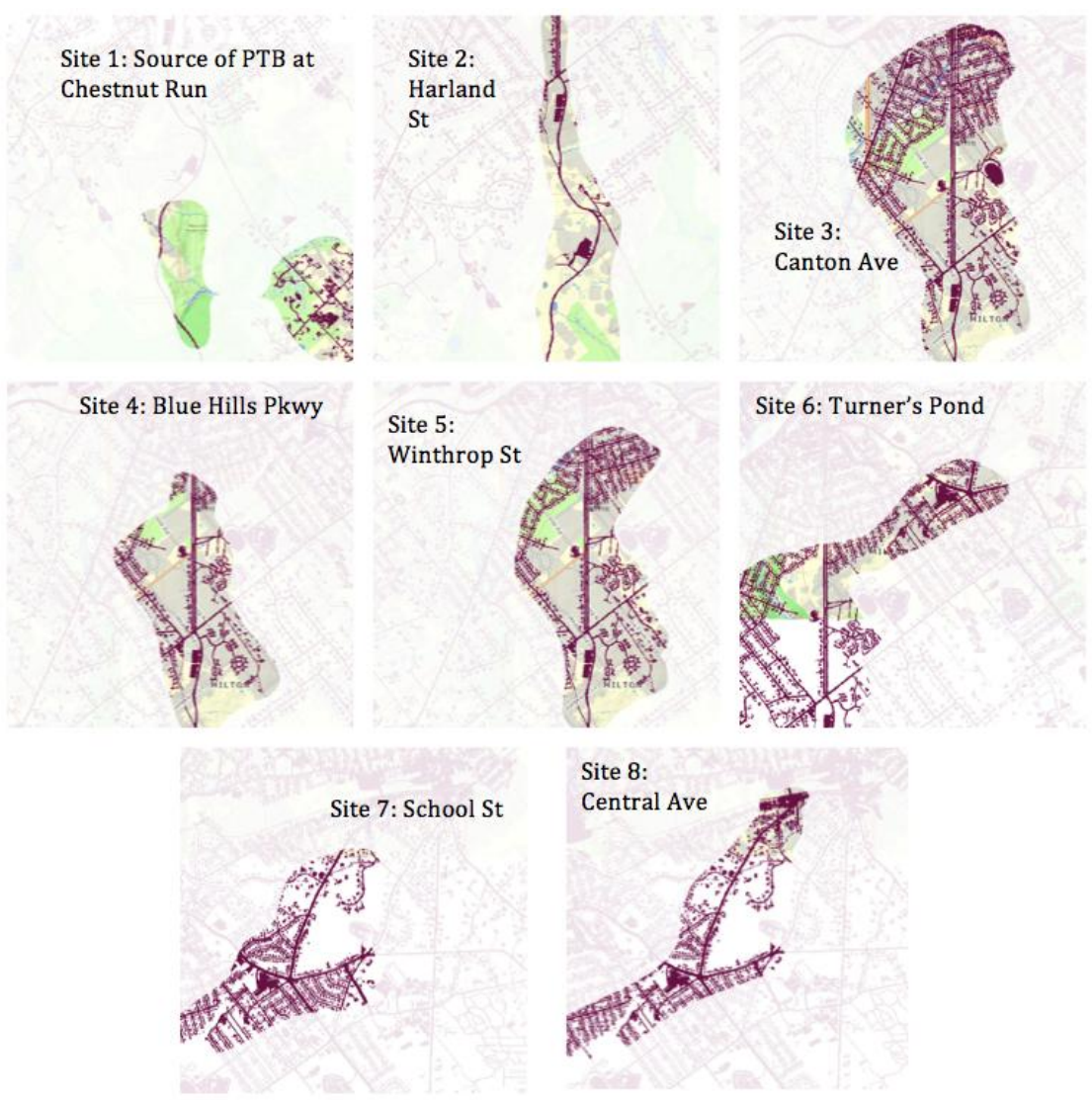

Figure3. Watershed for sample site within JPEG file for each location. These are the partial areas only. Accurate site areas for watershed and impervious surface data are the sum of all previous areas in all previous sites added to the current site's areas. The purple forms are the impervious surfaces that Oliver provided.

\section{RESULTS}

Figure 4 shows that $11.21 \%$ of the watershed at the source of Pine Tree Brook at Chestnut Run was covered by impervious surfaces. $17.67 \%$ of the watershed at the Harland Street location was covered by impervious surfaces.

Figure 5 shows that $-58 \mathrm{mg} / \mathrm{L}$ of TDS can be found at the source of Pine Tree Brook at Chestnut Run. The Blue Hills Parkway location displayed a $405 \mathrm{mg} / \mathrm{L}$ TDS reading. The end of Pine Tree Brook at Central Avenue displayed a $383 \mathrm{mg} / \mathrm{L}$ TDS reading.

Figure 6 shows that a $11.21 \%$ impervious surface cover corresponds with a $-58 \mathrm{mg} / \mathrm{L}$ TDS reading. The $15.65 \%$ impervious surface cover pairs with the $199 \mathrm{mg} / \mathrm{L}$ TDS reading. The $16.83 \%$ impervious surface cover corresponds with the $383 \mathrm{mg} / \mathrm{L}$ TDS measurement. The linear trend line fitted to the data is positive. The $17.67 \%$ impervious surface cover corresponds with a $154 \mathrm{mg} / \mathrm{L}$ TDS reading.

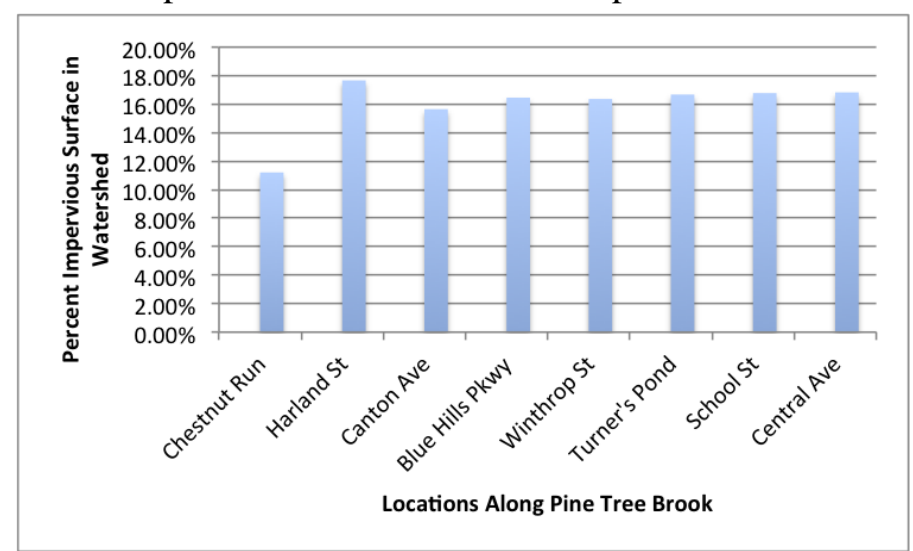

Figure4. Bar graph of percent impervious surface coverage in the watershed for each of the eight sites along Pine Tree Brook. Sites are listed left to right from the source of the brook at Chestnut Run to the end of the brook at Central Avenue. Sites are listed left to right denoting sites that go downstream from the source of the brook. 
Jason Kong \& Matthew Bingham "Total Dissolved Solids in Water as a Function of Growing Percent Impervious Surface Cover in the Watershed Suggest Pitfalls in Growth of Infrastructure?"

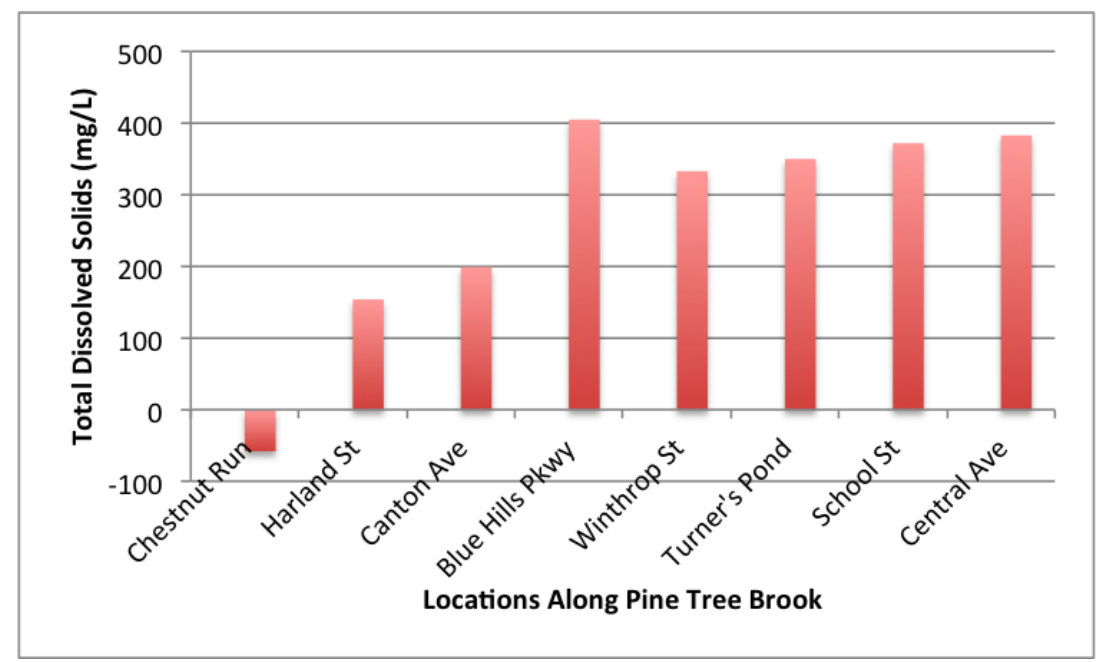

Figure5. Bar graph of TDS for eight sites along Pine Tree Brook. Sites are listed left to right from the source of the brook at Chestnut Run to the end of the brook at Central Avenue. Sites are listed left to right denoting sites that go downstream from the source of the brook.

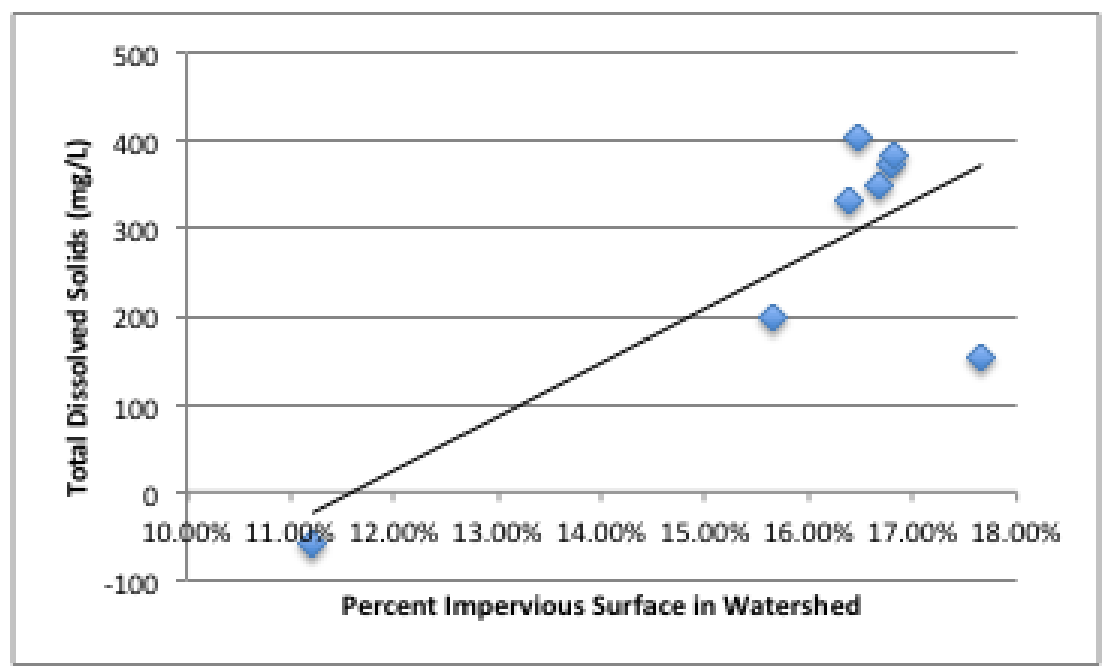

Figure6. Scatterplot of percent impervious surface in watershed vs. total dissolved solids. The eight data points represent the eight pairs of data recorded for each sampling site. The trend line chosen that best fits the data is linear. The $x$-axis has a modified minimum and maximum value range to provide a close perspective on the data.

\section{DISCUSSION}

To better understand the relationships between impervious surface cover and water quality, we first must consider how each separate set of data changes spatially. The data suggest that the percentages of impervious surface cover for the eight sites along Pine Tree Brook experience a slight positive trend (see Fig. 4). The largest increase in impervious surface percentage exists between the source of Pine Tree Brook at Chestnut Run in the Blue Hills and the Harland Street location. Because there exists a gradient of infrastructure between the source of Pine Tree Brook in the Blue Hills and the end of the brook in a medium-density residential area in the town of Milton, the positive trend can be explained by the fact that we had traveled from a fairly rural location in the woods to a location where there were a lot of buildings, roads, and sidewalks.

The Pine Tree Brook TDS measurements experience a moderate positive trend (see Fig. 5). The TDS reading increases from $-58 \mathrm{mg} / \mathrm{L}$ at the source of Pine Tree Brook, Chestnut Run, to $383 \mathrm{mg} / \mathrm{L}$ at the end of Pine Tree Brook, Central Avenue. Figure 5 contains one slight inconsistency. The maximum value of $405 \mathrm{mg} / \mathrm{L}$ TDS is found at the Blue Hills Parkway sampling location. During the day of fieldwork, the Blue Hills Parkway location exhibited road construction in progress. The oils, dirt, and other pollutants that characterize a construction project may have contributed more contaminants to the water in that location than usual. Though a more careful calibration of the conductivity sensor would be preferable, the negative TDS reading of $-58 \mathrm{mg} / \mathrm{L}$ is negligible because it is essentially 0 
Jason Kong \& Matthew Bingham "Total Dissolved Solids in Water as a Function of Growing Percent Impervious Surface Cover in the Watershed Suggest Pitfalls in Growth of Infrastructure?"

$\mathrm{mg} / \mathrm{L}$. Contrary to dense residential areas, rural areas are not exposed to as much contaminants because there are not that many cars, factories, or people that can pollute the water with chemicals or other harmful substances. This common knowledge can explain why the data suggests an increasing TDS reading.

The scatterplot data in Figure 6 ties together the relationship between percentage of impervious surface coverage and water quality at each location. The results of this experiment support our hypothesis that the TDS in the water will increase as the percentage of impervious surface cover increases. The data points experience a positive trend because the linear trend line that is fitted to the graph is positive. As the percentage of impervious surface coverage increased, the amount of TDS increased. This increase in TDS correlates with a decrease in water quality and an increase in stream pollution. This trend can be explained by how impervious surfaces do not allow water to infiltrate the soil. The increased flow of water carries more contaminants into the streams and bodies of water surrounding the impervious surfaces. In the case of this experiment, the increase in TDS as we move downstream from the source of Pine Tree Brook can also be explained by the difference in the amount of people and pollution-causing industrial factors between a rural area and a town. With more cars and manufacturing centered in the town, heightened TDS levels towards the end of Pine Tree Brook are expected. More pollutants are being produced in the town than in the rural area at the source of the brook.

The data suggest the direct relationship between the increased percentage of impervious surface cover and the increased amount of TDS in the water. What the data suggests to be true can be explained by a chain of events. As we moved from the source of the PTB in the Blue Hills, the surrounding area become more industrialized and surrounded by infrastructure. This gradient in infrastructure contributed to the increased impervious surface coverage and pollutant-causing agents, which leads to an increased TDS measurement of the sample location.

Though our data supports our hypothesis that TDS levels in the water will increase as the percentage of impervious surface coverage in the watershed increases, there exists some amount of uncertainty in the drawing of the watershed areas for each sampling site. Because the lines were drawn with the best judgment of a human, human error and bias could potentially harm the legitimacy of the data and the accuracy of the marked watersheds. To address this source of uncertainty, computer software that simulates the flow of water in areas with varying topography should be used to more accurately measure the behavior of the storm water run off that ends up in the stream. As a result, a more accurate representation of the watershed for each location could be achieved. Another source of uncertainty exists within the characteristics of the study site. Though the gradient of infrastructure and pollution-causing agents have significance in explaining why there is an increase in the percentage of impervious surface cover and TDS, they can also serve to confound the relationship between the percentage of impervious surface cover and water quality. For example, the heightened TDS readings in the more downstream locations may only be because there are more pollution-causing agents like people, factories, and cars and not just because of the impervious surface coverage. Likewise, the lower TDS readings in the more upstream locations could be only because of the lack of pollutioncausing agents because less cars and people are active in those areas. To attend to this uncertainty, we should sample a location that has an even number of pollution-causing agents throughout the study site but still a gradient of infrastructure between the beginning and end of the stream. Then, then we can isolate how the increasing impervious surface cover affects the water quality.

For future work with regards to this experiment, we should repeat the experiment in areas that have a larger gradient in infrastructure and, subsequently, the percentage of impervious surface cover so that the relationship between the percentage of impervious surface cover and water quality can be more defined. In future iterations of the same experiment, more emphasis can be place on the content of the water sampled. Dissolved oxygen, turbidity, and salinity can be measured concurrently with TDS to provide an even stronger understanding of the specific substances that contribute to the water quality. After determining the specific harmful substances in the water, measures can be taken by the government to regulate the proliferation of those substances. Future experiments should also be done after varying amounts of rainfall. In cases of heavy rainfall, even soil can display characteristics of an impervious surface because the water retention capacity of the soil can been filled and the soil cannot absorb any more water. Also, if the percolation of rainwater cannot keep up with the absorption rate, the water will also run off as if the soil is an impervious surface. 
Jason Kong \& Matthew Bingham "Total Dissolved Solids in Water as a Function of Growing Percent Impervious Surface Cover in the Watershed Suggest Pitfalls in Growth of Infrastructure?"

Despite the uncertainty in the demarcation of the watershed and the confounding variable of pollution-causing agents, this experiment displays a moderately positive trend between impervious surface cover and water contamination. These results suggest the need to strengthen the experimental methodology in order provide more defined evidence to argue that the growing global infrastructure contributes negatively to the quality of the water that society, animals, and plants rely on.

\section{REFERENCES}

[1] Beckwith, D. (2007). Evaluation of Rainfall-Runoff Relationships to Develop Stormwater Reduction Strategies in South Coast Watersheds. Retrieved January 22, 2017, from http://www.esm.ucsb.edu/research/documents/StormwaterBrief.pdf

[2] Center for Watershed Protection. (1998). Impervious Surfaces \& Water Quality. Retrieved January 22, 2017, from http://www.pwconserve.org/issues/watersheds/stormwater/ impervious. htm

[3] Clean Water Education Partnership. (2003). Clean Water Begins with You and Me. Retrieved January 22, 2017, from http://www.nccwep.org/stormwater/stormwater101/runoff.php

[4] Hill, J. (2009). The Environmental Impact of Roads. Retrieved January 22, 2017, from http://www.environmentalscience.org/roads

[5] Oram, B. (2014). Total Dissolved Solids and Water Quality. Retrieved January 22, 2017, from http://www.water-research.net/index.php/water-treatment/tools/total-dissolved-solids

[6] Polycarpou, L. (2010). No More Pavement! The Problem of Impervious Surfaces. Retrieved January 22, 2017, from http://blogs.ei.columbia.edu/2010/07/13/no-more-pavement-the-problem.

\section{AUTHOR'S BIOGRAPHY}

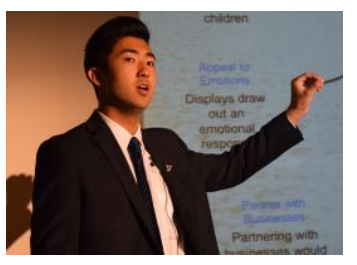

Jason Kong, is a high-school senior currently attending Milton Academy in Milton, MA, USA. As the head of his institution's sustainability board, he has spearheaded an environmentally oriented mindset within my institution's community through performing initiatives such as implementing school-wide waste regulation rules, establishing composting within buildings, harnessing solar energy, and conserving energy and water through eco-friendly washrooms and lighting. To pursue his passion in environmental policy, environmental anthropology, and advocacy, he conducted intensive ethnographic research under mentors Dr. Jenny Cook-Gumperz and Sara A. Cooley, MA as part of the prestigious Research Mentorship Program at the University of California, Santa Barbara. 